in vivo $33: 263-269(2019)$

doi:10.21873/invivo.11470

\title{
Risk Factors for Recurrence of Malignant Phyllodes Tumors of the Breast
}

\author{
HAE JIN PARK ${ }^{1 *}$, HAN SUK RYU ${ }^{2 *}$, KYUBO KIM $^{3}$, KYUNG HWAN SHIN ${ }^{4}$, \\ WONSHIK HAN ${ }^{5}$ and DONG-YOUNG NOH ${ }^{5}$

\begin{abstract}
${ }^{1}$ Department of Radiation Oncology, Hanyang University College of Medicine, Seoul, Republic of Korea;
${ }^{2}$ Department of Pathology, Seoul National University College of Medicine, Seoul, Republic of Korea;

${ }^{5}$ Department of Surgery, Seoul National University College of Medicine, Seoul, Republic of Korea
\end{abstract} \\ ${ }^{3}$ Department of Radiation Oncology, Ewha Womans University College of Medicine, Seoul, Republic of Korea; \\ ${ }^{4}$ Department of Radiation Oncology, Seoul National University College of Medicine, Seoul, Republic of Korea;
}

\begin{abstract}
Background/Aim: In this study, the treatment outcome and risk factors for recurrence in patients undergoing surgery with or without adjuvant radiotherapy (RT) for malignant phyllodes tumors of the breast (MPTB) were analyzed. Patients and Methods: Forty-three patients $(61.4 \%)$ underwent breast-conserving surgery (BCS) and 27 (38.6\%) underwent mastectomy. Fifteen patients (21.4\%) received adjuvant $R T$. Results: With a median follow-up of 76 months, the 7-year local control (LC), distant metastasisfree survival (DMFS), disease-free survival (DFS), and cause-specific survival (CSS) rates were 90.7\%, $85.2 \%$, $80.3 \%$, and $87.1 \%$, respectively. Either the extent of surgery or treatment with adjuvant RT did not affect the outcomes. On multivariate analysis, the presence of tumor necrosis was associated with inferior DFS ( $p=0.017)$, while infiltrative tumor border showed a marginal significance $(p=0.078)$. When stratified using these two adverse pathological features, the 7-year DFS rates were 100\%, 54.9\%, and $55.6 \%$ in patients with 0,1 , and 2 risk factors, respectively
\end{abstract}

This article is freely accessible online.

*These two Authors contributed equally to this study.

Correspondence to: Kyubo Kim, MD, Ph.D., Department of Radiation Oncology, Ewha Womans University College of Medicine, 1071, Anyangcheon-ro, Yangcheon-gu, Seoul 07985, Republic of Korea. Tel: +82 226505334, Fax: +82 226540363, e-mail: kyubokim.ro@gmail.com; Kyung Hwan Shin, MD, Ph.D., Department of Radiation Oncology, Seoul National University College of Medicine, 101 Daehak-ro, Jongno-gu, Seoul 03080, Republic of Korea. Tel: +82 220722524, Fax: +82 27653317, e-mail: radiat@snu.ac.kr

Key Words: Breast neoplasm, malignant phyllodes tumor, pathological feature, radiotherapy.
( $p=0.002)$. Conclusion: MPTB patients undergoing surgery with or without adjuvant RT had a favorable outcome. Although there was no local recurrence in patients treated with adjuvant $R T$, the effect of adjuvant $R T$ failed to reach a statistical significance. Risk-grouping based on pathological features might help design a clinical trial for MPTB.

Phyllodes tumors are rare fibroepithelial lesions that account for less than $1 \%$ of all primary breast neoplasms $(1,2)$. In 1981, the World Health Organization (WHO) adopted the term phyllodes tumors and subclassified them into benign, borderline, or malignant tumors according to histopathological characteristics such as stromal cellularity, cellular atypia, mitotic activity, stromal overgrowth, and tumor border $(3,4)$. The majority of these tumors are benign (35-64\%), and the rest are divided into borderline and malignant subtypes $(5,6)$. Although this classification has been helpful in predicting biological behavior, specific parameters that can define the likelihood of recurrence are not universally accepted (4). Certain studies have suggested the aforementioned histopathological features as prognostic factors $(6,7)$. On the other hand, other studies have reported that tumor size, necrosis, and adequacy of surgical margins are more important prognosticators (8-11).

Malignant phyllodes tumors of the breast (MPTB), on the contrary to the benign/borderline counterpart, are characterized by aggressive clinical features, propensity for local recurrence and capacity for distant metastasis (2). Given the high rate of local recurrence (LR) and their large tumor size, mastectomy has been the preferred surgical option for MPTB (12-15). Although breast-conserving surgery (BCS) +/- adjuvant radiotherapy (RT) is being frequently performed, there are only limited data on the effect of surgical extent or the benefit of adjuvant RT. In addition, most of the available studies analyzed all subtypes of phyllodes tumors altogether $(8-10,16)$ or borderline/malignant tumors combined $(7,17$, 18). Also, more importantly, there was no randomized trial for 
MPTB mainly due to its rarity. A few population-based studies and retrospective ones with a limited number of patients have reported the outcomes of MPTB exclusively $(9,15,19,20)$.

In this study, we analyzed the treatment outcomes of MPTB patients after surgical resection with or without adjuvant RT, and identified the risk factors of recurrence in these patients.

\section{Patients and Methods}

After Institutional Review Board approval, the medical records of patients with phyllodes tumor of the breast who underwent surgical resection at Seoul National University Hospital between December 1991 and January 2014 were retrospectively reviewed. Among them, pathologically diagnosed malignant subtypes were classified according to the WHO classification. Patients with coexisting breast cancer, either invasive carcinoma or carcinoma in situ, or with a previous breast cancer history were excluded from the analysis. A total of 70 patients were eligible for this study.

Clinico-pathological information was collected; it included age at diagnosis, tumor size, type of surgery, nodal examination, adjuvant RT, and pathologic features such as resection margin status, tumor necrosis, stromal cellularity, nuclear atypia, mitotic activity, and tumor border. Tumor on inked margin was defined as R1 resection. All available slides were re-evaluated by a single pathologist who was blind to the clinical data, according to WHO guidelines (21).

The primary outcome of interest was LR, defined as tumor recurrence in the ipsilateral breast or chest wall. Distant metastasis (DM), any recurrence (LR or DM), and death from MPTB were second outcomes of interest. These events (LR, DM, any recurrence, and death from MPTB) were used for survival analysis. Time to event was calculated as the date of surgical resection to the occurrence of each event. Actuarial local control (LC), distant metastasis-free survival (DMFS), disease-free survival (DFS), and cause-specific survival (CSS) rates were calculated according to the Kaplan-Meier method, and comparisons between groups were performed using logrank tests. A Cox proportional hazards model with backward stepwise method was used to identify correlations between outcomes and risk variables. $p$-Values $<0.05$ were considered statistically significant. Statistical analysis was performed using SPSS software (release 12.0.1. SPSS Inc., Chicago, IL, USA).

\section{Results}

Characteristics. The patient and tumor characteristics of all patients are summarized in Table I. The median age was 42 years (range $=19-70$ years). The median tumor size was $5.8 \mathrm{~cm}$ (range $=1.3-25 \mathrm{~cm})$. Forty-three patients $(61.4 \%)$ underwent BCS and 27 patients (38.6\%) underwent mastectomy. Seventeen patients had axillary nodal examination, and one showed nodal metastasis. Fifteen patients $(21.4 \%)$ were given adjuvant RT; 9 patients $(20.1 \%)$ after BCS and 6 patients (22.2\%) after mastectomy. The median radiation dose was 54.9 Gy (range=50.4-60 Gy). Three patients were treated with adjuvant chemotherapy, including one patient with nodal metastasis. Six patients had involved resection margin after $\mathrm{BCS}$, and one of them was given adjuvant RT.
Table I. Patient, tumor, and treatment characteristics.

\begin{tabular}{|c|c|c|}
\hline Variables & No. of patients & $\%$ \\
\hline \multicolumn{3}{|l|}{ Age, years } \\
\hline$\leq 40$ & 29 & $41 \%$ \\
\hline$>40$ & 41 & $59 \%$ \\
\hline \multicolumn{3}{|l|}{ Tumor size } \\
\hline$\leq 2 \mathrm{~cm}$ & 6 & $9 \%$ \\
\hline $2<$ to $\leq 5 \mathrm{~cm}$ & 22 & $31 \%$ \\
\hline $5<$ to $\leq 10 \mathrm{~cm}$ & 26 & $37 \%$ \\
\hline $10<$ to $\leq 20 \mathrm{~cm}$ & 11 & $16 \%$ \\
\hline$>20 \mathrm{~cm}$ & 2 & $3 \%$ \\
\hline Unknown & 3 & $4 \%$ \\
\hline \multicolumn{3}{|l|}{ Type of surgery } \\
\hline $\mathrm{BCS}$ & 43 & $61 \%$ \\
\hline Mastectomy & 27 & $39 \%$ \\
\hline \multicolumn{3}{|l|}{ Nodal examination } \\
\hline Yes & 17 & $24 \%$ \\
\hline No & 53 & $76 \%$ \\
\hline \multicolumn{3}{|c|}{ Increased cellularity } \\
\hline Mild-moderate & 35 & $50 \%$ \\
\hline Marked & 21 & $30 \%$ \\
\hline Unknown & 14 & $20 \%$ \\
\hline \multicolumn{3}{|l|}{ No. of mitoses } \\
\hline$\leq 5 / \mathrm{HPF}$ & 7 & $10 \%$ \\
\hline$>5 / \mathrm{HPF}$ & 56 & $80 \%$ \\
\hline Unknown & 7 & $10 \%$ \\
\hline \multicolumn{3}{|l|}{ Nuclear atypia } \\
\hline Mild-moderate & 31 & $44 \%$ \\
\hline Marked & 26 & $37 \%$ \\
\hline Unknown & 13 & $19 \%$ \\
\hline \multicolumn{3}{|l|}{ Tumor border } \\
\hline Pushing & 24 & $34 \%$ \\
\hline Infiltrative & 26 & $37 \%$ \\
\hline Unknown & 20 & $29 \%$ \\
\hline \multicolumn{3}{|c|}{ Stromal overgrowth } \\
\hline Absent & 17 & $24 \%$ \\
\hline Present & 36 & $51 \%$ \\
\hline Unknown & 17 & $24 \%$ \\
\hline \multicolumn{3}{|l|}{ Tumor Necrosis } \\
\hline Absent & 33 & $47 \%$ \\
\hline Present & 15 & $21 \%$ \\
\hline Unknown & 22 & $31 \%$ \\
\hline \multicolumn{3}{|l|}{ Resection margin } \\
\hline Negative & 34 & $49 \%$ \\
\hline Close $(\leq 2 \mathrm{~mm})$ & 27 & $39 \%$ \\
\hline Positive & 6 & $9 \%$ \\
\hline Unknown & 3 & $4 \%$ \\
\hline \multicolumn{3}{|l|}{ Adjuvant RT } \\
\hline Yes & 15 & $21 \%$ \\
\hline No & 55 & $79 \%$ \\
\hline
\end{tabular}

BCS: Breast-conserving surgery; HPF: high-power field; RT: radiotherapy.

Treatment outcomes and patterns of failure. With a median follow-up of 76 months (range=7-216 months), the 7-year LC, DMFS, DFS, and CSS rates were 90.7\%, 85.2\%, 80.3\%, and $87.1 \%$, respectively. There were 6 LR's; 5 in BCS group and one in mastectomy group. The median time to LR was 
Table II. Univariate analyses for local control, distant metastasis-free survival, disease-free survival, and cause-specific survival.

\begin{tabular}{|c|c|c|c|c|c|c|c|c|c|}
\hline Variables & No. of pts & 7-yr LC (\%) & p-Value & 7-yr DMFS (\%) & $\mathrm{p}$-Value & 7-yr DFS (\%) & p-Value & 7-yr CSS (\%) & $\mathrm{p}$-Value \\
\hline Age (yr) & & & 0.218 & & 0.601 & & 0.767 & & 0.819 \\
\hline$\leq 40$ & 29 & 85.4 & & 88.4 & & 79.3 & & 88.2 & \\
\hline$>40$ & 41 & 95.1 & & 82.7 & & 81.2 & & 86.1 & \\
\hline Tumor size & & & 0.057 & & 0.532 & & 0.681 & & 0.699 \\
\hline$\leq 5 \mathrm{~cm}$ & 28 & 100 & & 90.4 & & 75.5 & & 91.1 & \\
\hline$>5 \mathrm{~cm}$ & 39 & 78.9 & & 86.1 & & 86.1 & & 88.9 & \\
\hline Type of surgery & & & 0.209 & & 0.265 & & 0.629 & & 0.128 \\
\hline BCS & 43 & 90.0 & & 88.7 & & 85.2 & & 91.8 & \\
\hline Mastectomy & 27 & 90.9 & & 79.5 & & 72.3 & & 79.9 & \\
\hline Increased cellularity & & & 0.037 & & 0.030 & & 0.008 & & 0.084 \\
\hline Mild-moderate & 35 & 94.1 & & 94.1 & & 88.6 & & 94.1 & \\
\hline Marked & 21 & 81.8 & & 68.6 & & 63.7 & & 85.7 & \\
\hline No. of mitoses & & & 0.946 & & 0.998 & & 0.484 & & 0.909 \\
\hline$\leq 5$ & 7 & 85.7 & & 80.0 & & 85.7 & & 80.0 & \\
\hline$>5$ & 56 & 89.7 & & 83.7 & & 76.8 & & 86.0 & \\
\hline Nuclear atypia & & & 0.532 & & 0.395 & & 0.613 & & 0.250 \\
\hline Mild-moderate & 31 & 86.4 & & 89.1 & & 80.2 & & 92.2 & \\
\hline Marked & 26 & 92.9 & & 84.0 & & 78.0 & & 84.6 & \\
\hline Tumor border & & & 0.014 & & 0.196 & & 0.025 & & 0.072 \\
\hline Pushing & 24 & 100 & & 91.3 & & 91.3 & & 95.8 & \\
\hline Infiltrative & 26 & 63.7 & & 75.6 & & 52.8 & & 77.2 & \\
\hline Stromal overgrowth & & & 0.781 & & 0.217 & & 0.303 & & 0.266 \\
\hline Absent & 17 & 91.7 & & 94.1 & & 86.3 & & 93.8 & \\
\hline Present & 36 & 87.5 & & 76.6 & & 69.9 & & 80.1 & \\
\hline Tumor necrosis & & & 0.977 & & 0.007 & & 0.012 & & 0.027 \\
\hline Absent & 33 & 85.6 & & 92.1 & & 82.7 & & 92.7 & \\
\hline Present & 15 & 93.3 & & 65.2 & & 59.3 & & 72.0 & \\
\hline Resection margin & & & 0.000 & & 0.141 & & 0.003 & & 0.074 \\
\hline Uninvolved & 61 & 94.1 & & 87.4 & & 82.1 & & 89.4 & \\
\hline Involved & 6 & 50.0 & & 50.0 & & 50.0 & & 50.0 & \\
\hline Adjuvant RT & & & 0.237 & & 0.865 & & 0.684 & & 0.603 \\
\hline Yes & 15 & 100 & & 86.2 & & 86.2 & & 86.0 & \\
\hline No & 55 & 88.3 & & 85.5 & & 79.1 & & 92.9 & \\
\hline
\end{tabular}

*Patients with unknown information were not included on univariate analyses. LC: Local control; DMFS: distant metastasis-free survival; DFS: disease-free survival; CSS: cause-specific survival; BCS: breast-conserving surgery; RT: radiotherapy.

25 months (range=5-201 months). All LR's developed in patients not receiving adjuvant RT. Among 6 patients with involved resection margin, 4 experienced LR. One patient treated with adjuvant $\mathrm{RT}$ after $\mathrm{R} 1$ resection remained disease-free at the time of analysis ( 80 months from the date of surgical resection). DM occurred in 9 patients, and the site of metastasis was lung in 7 patients, brain in two, bone in one, and soft tissue in one. Two patients experienced LR and subsequent DM, which occurred 37 and 45 months apart. A total of 9 patients were dead at the time of analysis, and 8 of them died of DM of MPTB. The median time from the date of surgical resection to cause-specific death was 28 months. Prognostic factors affecting recurrences. The results of univariate analyses of LC, DMFS, DFS, and CSS rates are presented in Table II. The extent of surgery (BCS vs. mastectomy) did not affect LC ( $p=0.209$, Figure 1A), DMFS $(p=0.265)$, DFS $(p=0.629)$, and CSS $(p=0.128)$. Of note, there was no LR in patients treated with adjuvant RT (Figure 1B). Nevertheless, adjuvant RT did not show improvement in terms of LC $(p=0.237)$, DMFS $(p=0.865)$, DFS $(p=0.684)$, and CSS $(p=0.603)$. Univariate analyses of pathologic variables for LC revealed that increased cellularity, infiltrative tumor border, and involved surgical margin were significantly associated with inferior LC $(p=0.037,0.014$, and 0.001 , respectively). A tumor size $>5 \mathrm{~cm}$ was marginally associated with inferior LC $(p=0.057)$. Univariate analyses for DMFS revealed that increased cellularity and the presence of tumor necrosis were independent risk factors for inferior DMFS ( $p=0.030$ and 0.007 , respectively). As for DFS, increased cellularity, infiltrative tumor border, the presence of tumor necrosis, and involved surgical margin were significantly associated with inferior DFS $(p=0.008,0.025,0.012$, and 
A

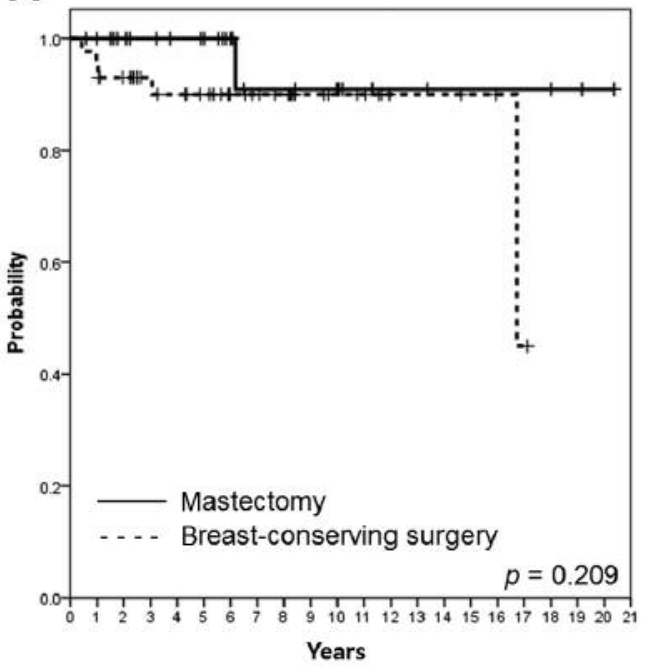

B

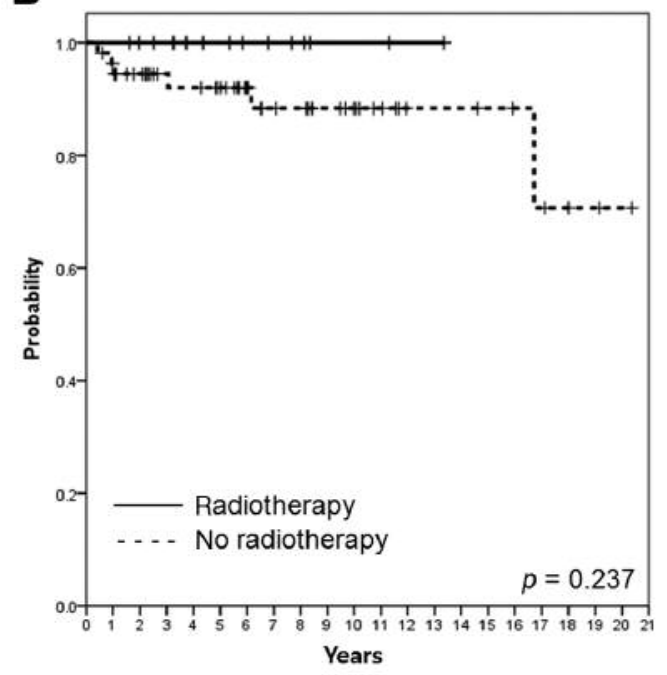

Figure 1. Probability of local control according to (A) type of surgery and (B) adjuvant radiotherapy.

A

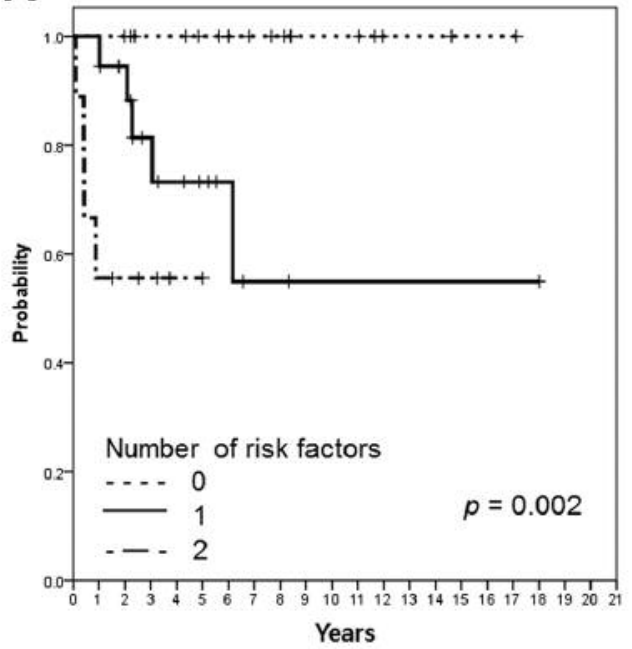

B

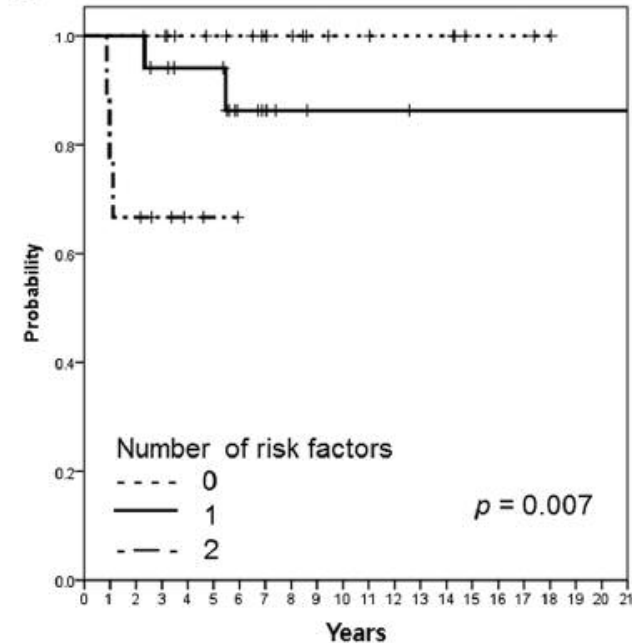

Figure 2. Disease-free survival (A), and cause-specific survival curves (B) according to increasing number of risk factors.

0.003 , respectively). Also, the presence of tumor necrosis was the only risk factor for inferior CSS $(p=0.027)$, although increased cellularity, infiltrative tumor border, and involved surgical margin were marginally significant $(p=0.084,0.072$, and 0.074 , respectively).

Multivariate analyses were performed incorporating variables with $p$-values $\leq 0.25$ on univariate analyses (22). The presence of tumor necrosis was found to be correlated significantly with inferior DMFS, DFS, and CSS $(p=0.006$,
Table III. Survival rates at 7-year according to increasing number of risk factors.

\begin{tabular}{lccc}
\hline $\begin{array}{l}\text { No. of risk } \\
\text { factors }\end{array}$ & $\begin{array}{l}\text { No. of } \\
\text { patients }\end{array}$ & $\begin{array}{c}7 \text {-yr DFS } \\
(p=0.002)\end{array}$ & $\begin{array}{c}7-\mathrm{yr} \mathrm{CSS} \\
(p=0.007)\end{array}$ \\
\hline 0 & 18 & $100 \%$ & $100 \%$ \\
1 & 18 & $54.9 \%$ & $86.3 \%$ \\
2 & 9 & $55.6 \%$ & $66.7 \%$ \\
\hline
\end{tabular}

DFS: Disease-free survival; CSS: cause-specific survival. 
Table IV. Reported risk factors predicting inferior local control, distant metastasis-free survival, disease-free survival, and cause-specific survival.

\begin{tabular}{|c|c|c|c|c|c|c|c|}
\hline Study & No. of pts & Database & $\%$ of RT & $\mathrm{LC}$ & DMFS & DFS & CSS \\
\hline Kapris et al. (23) & $34\left(48^{a}\right)$ & $\begin{array}{l}\text { A single } \\
\text { institution }\end{array}$ & $6.2 \%(3 / 48)$ & $\begin{array}{c}\text { Larger tumor, } \\
\text { involved resection } \\
\text { margin }\end{array}$ & $\begin{array}{c}\text { Larger tumor, } \\
\text { involved resection } \\
\text { margin }\end{array}$ & - & - \\
\hline Asoglu et al. (24) & 50 & $\begin{array}{l}\text { A single } \\
\text { institution }\end{array}$ & $0.04 \%(2 / 50)$ & $\begin{array}{c}\text { Stromal } \\
\text { overgrowth, } \\
\text { larger tumor, } \\
\text { margin }<1 \mathrm{~cm}\end{array}$ & $\begin{array}{c}\text { Stromal } \\
\text { overgrowth }\end{array}$ & - & $\begin{array}{c}\text { Stromal } \\
\text { overgrowth }^{\mathrm{b}}\end{array}$ \\
\hline Macdonald et al. (15) & 821 & $\begin{array}{c}\text { SEER } \\
\text { database }\end{array}$ & $9.2 \%(76 / 821)$ & - & - & - & $\begin{array}{l}\text { Older age, } \\
\text { adjuvant RT }\end{array}$ \\
\hline Pezner et al. (11) & 478 & $\begin{array}{l}\text { National } \\
\text { Oncology } \\
\text { Database }\end{array}$ & $0 \%$ & $\begin{array}{l}\text { Larger tumor } \\
(>2 \mathrm{~cm} \text { for } \mathrm{BCS} \\
>10 \mathrm{~cm} \text { for } \\
\text { mastectomy })\end{array}$ & - & - & $\begin{array}{l}\text { Older age, } \\
\text { larger tumor }\end{array}$ \\
\hline Onkendi et al. (7) & $52\left(67^{a}\right)$ & $\begin{array}{l}\text { A single } \\
\text { institution }\end{array}$ & $6.0 \%(4 / 67)$ & - & - & $\begin{array}{c}\text { Larger tumor, } \\
\text { high cellularity, } \\
\text { mitosis } \geq 10 / 10 \text { HPFs, } \\
\text { stromal overgrowth }\end{array}$ & $\begin{array}{l}\text { Mitosis } \geq 10 / 10 \\
\text { HPFs, stromal } \\
\text { overgrowth, } \\
\text { high cellularity }\end{array}$ \\
\hline Mitus et al. (9) & 70 & $\begin{array}{l}\text { A single } \\
\text { institution }\end{array}$ & $8.6 \%(6 / 70)$ & - & - & None & - \\
\hline This study & 70 & $\begin{array}{l}\text { A single } \\
\text { institution }\end{array}$ & $21.4 \%(15 / 70)$ & - & $\begin{array}{l}\text { Tumor necrosis, } \\
\text { infiltrative } \\
\text { tumor border }\end{array}$ & Tumor necrosis & Tumor necrosis \\
\hline
\end{tabular}

aSum of borderline and malignant subtype; bfor overall survival; ${ }^{c}$ marginally significant. LC: Local control; DMFS: distant metastasis-free survival; DFS: disease-free survival; CSS: cause-specific survival; BCS: breast-conserving surgery; HPF: high-power field; RT: radiotherapy.

0.017 , and 0.030 , respectively). Infiltrative tumor border was marginally associated with DFS $(p=0.078)$. Any of these variables was not associated with LC.

To identify patients at a higher risk of recurrence, risk stratification was performed according to the number of adverse clinicopathologic features associated with either local or distant recurrence. The presence of tumor necrosis $(=0.017)$ and infiltrative tumor border $(p=0.078)$ were selected based on multivariate analyses for DFS. The DFS and CSS rates were significantly different among the three groups ( $p=0.002$ and 0.007, respectively) (Table III and Figure 2).

\section{Discussion}

The optimal local treatment for MPTB is controversial. Given the large tumor size and the high LR rate, a significant number of patients have been treated with mastectomy. However, according to the recent Surveillance, Epidemiology, and End Results (SEER) analysis (20), 58\% of patients are treated with BCS, and adjuvant RT is offered in $16 \%$ of patients after mastectomy and $11 \%$ after BCS. In the current study, $61.4 \%$ of patients underwent BCS, and in $22.2 \%$ of patients adjuvant RT was given after mastectomy and in $20.1 \%$ after BCS. The present study showed that no LR occurred in patients treated with adjuvant RT regardless of the type of surgery (BCS or mastectomy). Even though statistical significance was not reached, possible benefit from adjuvant RT might be expected because this result may have been influenced by the different treatments the few patients received. Regarding the benefit of adjuvant RT in MPTB, Gnerlich et al. noted that adjuvant RT reduced LR (hazard ratio=0.43) via the National Cancer Data Base analysis (19). Belkacemi et al. also noted that adjuvant RT increased LC rate in borderline/malignant phyllodes tumors (15). A recent meta-analysis by Zeng et al. observed a similar reduction in LR by adjuvant RT (hazard ratio=0.43) in borderline/ malignant phyllodes tumors. However, according to the type of surgery, the improved LC was mainly seen in patients treated with BCS, not with mastectomy (17). Although there was no randomized trial on the benefit of adjuvant RT in MPTB, a multicenter prospective trial was conducted, evaluating the efficacy of adjuvant RT after BCS in borderline/malignant phyllodes tumors, and no LR was identified at a median follow-up of 56 months (18).

The capacity for DM as well as LR is a distinguishable feature of MPTB from benign/borderline phyllodes tumors. The reported DM rates of MPTB ranged from 14.3 to $27.0 \%$ (9), and the most frequent metastatic site was the lung. The results of our study were consistent with these observations; 9 out of 70 patients (12.9\%) developed DM, and 7 had lung 
metastasis. Whether LR is associated with DM or instigates DM is not clear. Kapiris et al. showed that 11 of 13 patients with DM had developed LR before the diagnosis of DM (23), and Asoglu et al. also showed that 8 of 13 patients with DM had a preceding LR (24). On the other hand, Mitus et al. reported DM in 10 of 70 patients, but no LR occurred among them (9). Similarly, only 2 patients experienced LR and subsequent DM in the present study.

The widely known risk factors predicting LR includes resection margin involvement, larger tumor size, and pathologic features (Table IV). Two studies, focused on MPTB, reported that involved resection margin was the predictor of LR; Kapris et al. found that tumor size and resection margin were associated with LR (23), and Asoglu et al. found that stromal overgrowth, tumor size, and resection margin were significant prognosticators for LR (24). However, the number of MPTB patients was only 34 in the former and 50 in the latter, respectively. The current study included 70 MPTB patients from a single institution, and available pathological slides were reviewed by a single pathologist although some information was still unavailable. On univariate analysis of LC, cellularity, tumor border, and resection margin status were significantly associated with LR, and the statistical significance of tumor size was marginal. As for DM, the aforementioned studies analyzing MPTB showed inconsistent results. The present study showed that the presence of tumor necrosis was the only independent risk factor for inferior DMFS. On the other hand, Kapris et al. reported that tumor size and resection margin were the principal determinants of LR as well as DM (23), and Asoglu et al. reported stromal overgrowth as the only significant predictor for DM (24). As for DFS, our study showed that the presence of tumor necrosis was an independent factor predicting inferior DFS, while infiltrative tumor border showed marginal significance. Meanwhile, Onkendi et al. conducted a retrospective analysis for borderline/malignant phyllodes tumors and noted that tumor size, mitosis, stromal overgrowth, and cellularity were predictive of DFS (7). Belkacemi et al. noted that mitosis, cellular atypia, stromal overgrowth, and tumor necrosis were predictive of DFS on univariate analysis, but the significance disappeared on multivariate analysis for all types of phyllodes tumors.

Although the current study showed an overall favorable LC (90.7\% at 7-year) and DMFS (85.2\% at 7-year), there might be a certain group of patients at higher risk of recurrence. We performed risk stratification according to 2 prognostic factors associated with either local or distant recurrences: presence of tumor necrosis and infiltrative tumor border. Patients with 2 risk factors showed a 7-year DFS rate of $55.6 \%$ and a 7year CSS rate of $66.7 \%$, whereas all patients with no risk factors survived without recurrence at 7 -year. Therefore, highrisk patients might need more intensified treatment, but the benefit of either adjuvant RT or systemic chemotherapy has not yet been demonstrated in the MPTB. Further studies are needed to elucidate the optimal treatment strategy for these patients, and the risk-grouping suggested here might be helpful in the design of clinical trials in the future.

The present study was retrospectively designed, and therefore, findings from this study should be interpreted with caution. Follow-up period was also relatively short considering the long natural history of phyllodes tumor of the breast. In addition, although all available slides were reviewed by a single pathologist, unknown pathologic features were still common. This might hamper an accurate analysis. Despite these limitations, the strength of our study is that it included exclusively an MPTB cohort, and not benign or borderline subtypes.

In conclusion, this study presented a favorable outcome in MPTB patients treated with surgical resection with or without adjuvant RT. Although there was no LR in patients receiving adjuvant RT, its benefit is still uncertain. Risk stratification was done based on adverse pathological features, and DFS and CSS were well separated according to the number of risk factors. This risk-grouping might help design a clinical trial for MPTB in the future.

\section{References}

1 Moffat CJ, Pinder SE, Dixon AR, Elston CW, Blamey RW and Ellis IO: Phyllodes tumours of the breast: A clinicopathological review of thirty-two cases. Histopathology 27(3): 205-218, 1995.

2 Parker SJ and Harries SA: Phyllodes tumours. Postgrad Med J 77(909): 428-435, 2001.

3 The World Health Organization: Histological typing of breast tumors. Neoplasma 30(1): 113-123, 1983.

4 Tan PH, Jayabaskar T, Chuah KL, Lee HY, Tan Y, Hilmy M, Hung H, Selvarajan S and Bay BH: Phyllodes tumors of the breast: The role of pathologic parameters. Am J Clin Pathol 123(4): 529-540, 2005.

5 Salvadori B, Cusumano F, Del Bo R, Delledonne V, Grassi M, Rovini D, Saccozzi R, Andreola S and Clemente C: Surgical treatment of phyllodes tumors of the breast. Cancer 63(12): 2532-2536, 1989.

6 Reinfuss M, Mitus J, Duda K, Stelmach A, Rys J and Smolak K: The treatment and prognosis of patients with phyllodes tumor of the breast: An analysis of 170 cases. Cancer 77(5): 910-916, 1996.

7 Onkendi EO, Jimenez RE, Spears GM, Harmsen WS, Ballman $\mathrm{KV}$ and Hieken TJ: Surgical treatment of borderline and malignant phyllodes tumors: The effect of the extent of resection and tumor characteristics on patient outcome. Ann Surg Oncol 21(10): 3304-3309, 2014.

8 Yom CK, Han W, Kim SW, Park SY, Park IA and Noh DY: Reappraisal of conventional risk stratification for local recurrence based on clinical outcomes in 285 resected phyllodes tumors of the breast. Ann Surg Oncol 22(9): 2912-2918, 2015.

9 Mitus J, Reinfuss M, Mitus JW, Jakubowicz J, Blecharz P, Wysocki WM and Skotnicki P: Malignant phyllodes tumor of the breast: Treatment and prognosis. Breast J 20(6): 639-644, 2014. 
10 Jang JH, Choi MY, Lee SK, Kim S, Kim J, Lee J, Jung SP, Choe JH, Kim JH, Kim JS, Cho EY, Lee JE, Nam SJ and Yang JH: Clinicopathologic risk factors for the local recurrence of phyllodes tumors of the breast. Ann Surg Oncol 19(8): 26122617, 2012.

11 Pezner RD, Schultheiss TE and Paz IB: Malignant phyllodes tumor of the breast: Local control rates with surgery alone. Int J Radiat Oncol Biol Phys 71(3): 710-713, 2008.

12 Dyer NH, Bridger JE and Taylor RS: Cystosarcoma phylloides. Br J Surg 53(5): 450-455, 1966.

13 Maier WP, Rosemond GP, Wittenberg P and Tassoni EM: Cytosarcoma phyllodes mammae. Oncology 22(2): 145-158, 1968.

14 Pandey M, Mathew A, Kattoor J, Abraham EK, Mathew BS, Rajan B and Nair KM: Malignant phyllodes tumor. Breast J 7(6): 411-416, 2001.

15 Macdonald OK, Lee CM, Tward JD, Chappel CD and Gaffney DK: Malignant phyllodes tumor of the female breast: Association of primary therapy with cause-specific survival from the surveillance, epidemiology, and end results (seer) program. Cancer 107(9): 2127-2133, 2006.

16 Belkacemi Y, Bousquet G, Marsiglia H, Ray-Coquard I, Magne N, Malard Y, Lacroix M, Gutierrez C, Senkus E, Christie D, Drumea K, Lagneau E, Kadish SP, Scandolaro L, Azria D and Ozsahin M: Phyllodes tumor of the breast. Int J Radiat Oncol Biol Phys 70(2): 492-500, 2008.

17 Zeng S, Zhang X, Yang D, Wang X and Ren G: Effects of adjuvant radiotherapy on borderline and malignant phyllodes tumors: A systematic review and meta-analysis. Mol Clin Oncol 3(3): 663-671, 2015.
18 Barth RJ Jr., Wells WA, Mitchell SE and Cole BF: A prospective, multi-institutional study of adjuvant radiotherapy after resection of malignant phyllodes tumors. Ann Surg Oncol 16(8): 2288-2294, 2009.

19 Gnerlich JL, Williams RT, Yao K, Jaskowiak N and Kulkarni SA: Utilization of radiotherapy for malignant phyllodes tumors: Analysis of the national cancer data base, 1998-2009. Ann Surg Oncol 21(4): 1222-1230, 2014.

$20 \mathrm{Kim}$ YJ and Kim K: Radiation therapy for malignant phyllodes tumor of the breast: An analysis of seer data. Breast 32: 26-32, 2017.

21 Lakhani SR: WHO classification of tumours of the breast. International Agency for Research on Cancer, 2012.

22 Hosmer Jr DW, Lemeshow S and Sturdivant RX: Applied logistic regression. John Wiley \& Sons, 2013.

23 Kapiris I, Nasiri N, A'Hern R, Healy V and Gui GP: Outcome and predictive factors of local recurrence and distant metastases following primary surgical treatment of high-grade malignant phyllodes tumours of the breast. Eur J Surg Oncol 27(8): 723-730, 2001.

24 Asoglu O, Ugurlu MM, Blanchard K, Grant CS, Reynolds C, Cha SS and Donohue JH: Risk factors for recurrence and death after primary surgical treatment of malignant phyllodes tumors. Ann Surg Oncol 11(11): 1011-1017, 2004.

Received October 20, 2018

Revised October 30, 2018

Accepted October 31, 2018 